\title{
TWO DIMENSIONAL THERMO-ELASTIC WAVES DUE TO DISTURBANCE PRODUCED BY AN IMPULSIVE HEAT NUCLEUS
}

\author{
BISWAJIT DATTA AND B. DAS
}

\begin{abstract}
This paper deals with a two-dimensional dynamical problem of thermoelasticity generating elastic waves due to disturbance produce by a periodic heat nucleus. Initially, a detailed discussion on the theoretical part of the problem ${ }^{[2]}$ is made without temperature, then the disturbance due to impulsive heat nucleus is considered.
\end{abstract}

\section{Intrduction}

In a paper, Eason, Fulton and Sneddon ${ }^{[3]}$ have dealt with the disturbances produced by the distribution of stresses in an infinite elastic solid when the time dependent body force act upon certain region of the body. Strain being small, the general solution of the equation of motion for any distribution of body force is derived by the Four-dimensional Fourrier transforms ${ }^{[4]}$ and thus the general solution is obtained. It is a typical two dimensional problem when the disturbance is generated due to time dependent impulsive heat nucleus.

\section{Method of Solution}

Equation of motion in two dimensions with density $\rho$ of the medium are ${ }^{[1]}$

$$
\left.\begin{array}{c}
\frac{\partial \tau^{x x}}{\partial x}+\frac{\partial \tau^{x y}}{\partial y}+\rho F^{x}=\rho f^{x} \\
\frac{\partial \tau^{x y}}{\partial x}+\frac{\partial \tau^{y y}}{\partial y}+\rho F^{y}=\rho f^{y}
\end{array}\right\}
$$

where $F^{x}, F^{y}$ denote components of body force at $(x, y)$.

The acceleration of the infinitisimal element centred at this point is denoted by $\left(f^{x}, f^{y}\right)$. If we introduce the displacement vector components $\left(v^{x}, v^{y}\right)$ at such a typi-

Received February 19, 2002; revised June 18, 2002.

2000 Mathematics Subject Classification. 74F05.

Key words and phrases. Thermal stress, strain, elastic waves, impulsive heat nucleus, Bessel function, Fourier transform. 
cal point, we have

$$
\left.\begin{array}{l}
f^{x}=\frac{\partial^{2} v^{x}}{\partial t^{2}}=c^{2} \frac{\partial^{2} v^{x}}{\partial \tau^{2}} \\
f^{y}=\frac{\partial^{2} v^{y}}{\partial t^{2}}=c^{2} \frac{\partial^{2} v^{y}}{\partial \tau^{2}}
\end{array}\right\}
$$

where $t$ denotes time, $c$ is some characteristic velocity and $\tau=c t$, is a space time coordinate determined by time. The equation of motion may, therefore, be written in the form ${ }^{[5]}$

$$
\left.\begin{array}{c}
\frac{\partial \tau^{x x}}{\partial x}+\frac{\partial \tau^{x y}}{\partial y}+\rho F^{x}=\rho c^{2} \frac{\partial^{2} v^{x}}{\partial \tau^{2}} \\
\frac{\partial \tau^{x y}}{\partial x}+\frac{\partial \tau^{y y}}{\partial y}+\rho F^{y}=\rho c^{2} \frac{\partial^{2} v^{y}}{\partial \tau^{2}}
\end{array}\right\}
$$

To solve these equation we introduce Fourier transform ${ }^{[4]}$ of each of the components of stress and displacement. We shall denote the Fourier transforms of a function $\phi$ by placing a bar over it, thus $\bar{\phi}$; in other word

$$
\left.\begin{array}{l}
\frac{1}{(2 \pi)^{\frac{3}{2}}} \int_{s_{3}} \phi\left(x_{1}, x_{2}, \tau\right) \exp \left\{i\left(x_{p} \xi_{p}+\omega \tau\right)\right\} d s=\bar{\phi}\left(\xi_{x}, \xi_{y}, \omega\right) \\
\frac{1}{(2 \pi)^{\frac{3}{2}}} \int_{s_{3}}\left(\frac{\partial \phi}{\partial x_{p}}, \frac{\partial^{2} \phi}{\partial \tau^{2}}\right) \exp \left\{i\left(x_{p} \xi_{p}+\omega \tau\right)\right\} d s=-\left(i \xi_{p}, \omega^{2}\right) \bar{\phi}
\end{array}\right\}
$$

where $p=1,2 ; d s=d x_{1} d x_{2} d \tau$ and $s_{3}$ denotes the entire $x_{1} x_{2} \tau$ plane. Applying (2) in (1), we get

$$
\left.\begin{array}{l}
i \xi_{x} \bar{\tau}^{x x}+i \xi_{y} \bar{\tau}^{x y}-\rho \bar{F}^{x}=\rho c^{2} \omega^{2} \bar{v}^{x} \\
i \xi_{x} \bar{\tau}^{x y}+i \xi_{y} \bar{\tau}^{y y}-\rho \bar{F}^{y}=\rho c^{2} \omega^{2} \bar{v}^{y}
\end{array}\right\}
$$

From stress-strain relations in two dimensional thermoelastic problem for a temperature distribuion $T^{[5]}$, we have

$$
\left.\begin{array}{rl}
\tau^{x x} & =E_{1}\left(\frac{\partial v^{x}}{\partial x}+\nu \frac{\partial v^{y}}{\partial y}-\alpha_{1} T\right) \\
\tau^{y y} & =E_{1}\left(\nu \frac{\partial v^{x}}{\partial x}+\frac{\partial v^{y}}{\partial y}-\alpha_{1} T\right) \\
\tau^{x y} & =\mu\left(\frac{\partial v^{x}}{\partial y}+\nu \frac{\partial v^{y}}{\partial x}\right)
\end{array}\right\}
$$

where $E_{1}=\frac{E}{1-\nu^{2}}, \alpha_{1}=(1+\nu) \alpha, \nu=\frac{E}{2(1+\nu)}, E$ and $\alpha$ denote Young's modulus and coefficient of linear thermal expansion.

Using (2) over (4) we get

$$
\left.\begin{array}{l}
\bar{\tau}^{x x}=-i E_{1}\left(\xi_{x} \bar{v}^{x}+\nu \xi_{y} \bar{v}^{y}-i \alpha_{1} \bar{T}\right) \\
\bar{\tau}^{y y}=-i E_{1}\left(\nu \xi_{x} \bar{v}^{x}+\xi_{y} \bar{v}^{y}-i \alpha_{1} \bar{T}\right) \\
\bar{\tau}^{x y}=-i \mu\left(\xi \bar{v}^{x}+\xi \bar{v}^{y}\right) .
\end{array}\right\}
$$


Substituting (5) in (3), and solving for $\bar{v}^{x}$ and $\bar{v}^{y}$ we get,

$$
\begin{aligned}
& \left.\begin{array}{rl}
\bar{v}^{x}= & \left\{\beta^{2}\left(\xi_{x}^{2}+\xi_{y}^{2}-\omega^{2}\right) \bar{F}^{x}-\left(\beta^{2}-1\right)\left(\xi_{x}^{2} \bar{F}^{x}+\xi_{y} \xi_{x} \bar{F}^{y}\right)\right. \\
& \left.+\frac{2 i c_{1}^{2}}{\beta^{2}}\left(\beta^{2}-1\right) \alpha \bar{T} \xi_{x}\left(\xi_{x}^{2}+\xi_{y}^{2}-\omega^{2}\right)\right\} \\
& \cdot\left\{c_{1}^{2}\left(\xi_{x}^{2}+\xi_{y}^{2}-\beta^{2} \omega^{2}\right)\left(\xi_{x}^{2}+\xi_{y}^{2}-\omega^{2}\right)\right\}^{-1}
\end{array}\right\} \\
& \bar{v}^{y}=\left\{\beta^{2}\left(\xi_{x}^{2}+\xi_{y}^{2}-\omega^{2}\right) \bar{F}^{y}-\left(\beta^{2}-1\right)\left(\xi_{x} \xi_{y} \bar{F}^{x}+\xi_{y}^{2} \bar{F}^{y}\right)\right. \\
& \left.+\frac{2 i c_{1}^{2}}{\beta^{2}}\left(\beta^{2}-1\right) \alpha \bar{T} \xi_{y}\left(\xi_{x}^{2}+\xi_{y}^{2}-\omega^{2}\right)\right\} \\
& \cdot\left\{c_{1}^{2}\left(\xi_{x}^{2}+\xi_{y}^{2}-\beta^{2} \omega^{2}\right)\left(\xi_{x}^{2}+\xi_{y}^{2}-\omega^{2}\right)\right\}^{-1}
\end{aligned}
$$

where $\beta^{2}=\frac{4(\lambda+\mu)}{\lambda+2 \mu} \cdot c_{1}^{2}=\frac{4 \mu(\lambda+\mu)}{\rho(\lambda+2 \mu)}$ is chosen for our characteristic velociry $c$. Now, we find from (5)

$$
\begin{aligned}
& \bar{\tau}^{x x}=-i \rho\left\{\beta^{2}\left(\xi_{x}^{2}+\xi_{y}^{2}-\omega^{2}\right)\left(\xi_{x} \bar{F}^{x}+\nu \xi_{y} \bar{F}^{y}\right)-\left(\beta^{2}-1\right)\left(\xi_{x}^{2}+\nu \xi_{y}^{2}\right)\left(\xi_{x} \bar{F}^{x}+\xi_{y} \bar{F}^{y}\right)\right. \\
& \left.+\frac{2 i c_{1}^{2}}{\beta^{2}}\left(\beta^{2}-1\right) \alpha \bar{T}\left(\xi_{x}^{2}+\xi_{y}^{2}-\beta^{2} \omega^{2}\right)\left[(\nu-1) \xi_{y}^{2}+\omega^{2}\right]\right\} \\
& \cdot\left\{\left(\xi_{x}^{2}+\xi_{y}^{2}-\beta^{2} \omega^{2}\right)\left(\xi_{x}^{2}+\xi_{y}^{2}-\omega^{2}\right)\right\}^{-1} \\
& \bar{\tau}^{y y}=-i \rho\left\{\beta^{2}\left(\xi_{x}^{2}+\xi_{y}^{2}-\omega^{2}\right)\left(\nu \xi_{x} \bar{F}^{x}+\xi_{y} \bar{F}^{y}\right)-\left(\beta^{2}-1\right)\left(\nu \xi_{x}^{2}+\xi_{y}^{2}\right)\left(\xi_{x} \bar{F}^{x}+\xi_{y} \bar{F}^{y}\right)\right. \\
& \left.+\frac{2 i c_{1}^{2}}{\beta^{2}}\left(\beta^{2}-1\right) \alpha \bar{T}\left(\xi_{x}^{2}+\xi_{y}^{2}-\beta^{2} \omega^{2}\right)\left[(\nu-1) \xi_{y}^{2}+\omega^{2}\right]\right\} \\
& \cdot\left\{\left(\xi_{x}^{2}+\xi_{y}^{2}-\beta^{2} \omega^{2}\right)\left(\xi_{x}^{2}+\xi_{y}^{2}-\omega^{2}\right)\right\}^{-1} \\
& \bar{\tau}^{x y}=\frac{-i \rho}{\beta^{2}}\left\{\beta^{2}\left(\xi_{x}^{2}+\xi_{y}^{2}-\omega^{2}\right)\left(\xi_{y} \bar{F}^{x}+\xi_{x} \bar{F}^{y}\right)-2\left(\beta^{2}-1\right) \xi_{x} \xi_{y}\left(\xi_{x} \bar{F}^{x}+\xi_{y} \bar{F}^{y}\right)\right. \\
& \left.+\frac{2 i c_{1}^{2}}{\beta^{2}}\left(\beta^{2}-1\right) \alpha \bar{T}\left(\xi_{x}^{2}+\xi_{y}^{2}\right)\left(\xi_{x}^{2}+\xi_{y}^{2}-\beta^{2} \omega^{2}\right)\right\} \\
& \cdot\left\{\left(\xi_{x}^{2}+\xi_{y}^{2}-\beta^{2} \omega^{2}\right)\left(\xi_{x}^{2}+\xi_{y}^{2}-\omega^{2}\right)\right\}^{-1}
\end{aligned}
$$

Applying Fourier inversion theorem ${ }^{[4]}$ over $(6)-(10)$, we have

$$
\left.\begin{array}{rl}
v^{x}= & \frac{1}{c_{1}^{2}(2 \pi)^{\frac{3}{2}}} \int_{W_{3}}\left\{\beta^{2}\left(\xi_{x}^{2}+\xi_{y}^{2}-\omega^{2}\right) \bar{F}^{x}-\left(\beta^{2}-1\right)\left(\xi_{x}^{2} \bar{F}^{x}+\xi_{x} \xi_{y} \bar{F}^{y}\right)\right. \\
& \left.+\frac{2 i c_{1}^{2}}{\beta^{2}}\left(\beta^{2}-1\right) \alpha \bar{T} \xi_{x}\left(\xi_{x}^{2}+\xi_{y}^{2}-\omega^{2}\right)\right\} \cdot \exp \left[-i\left(x \xi_{x}+y \xi_{y}+\omega \tau\right)\right] \\
& \cdot\left\{\left(\xi_{x}^{2}+\xi_{y}^{2}-\beta^{2} \omega^{2}\right)\left(\xi_{x}^{2}+\xi_{y}^{2}-\omega^{2}\right)\right\}^{-1} \cdot d W
\end{array}\right\}
$$




$$
\left.\begin{array}{rl}
v^{x}= & \frac{1}{c_{1}^{2}(2 \pi)^{\frac{3}{2}}} \int_{W_{3}}\left\{\beta^{2}\left(\xi_{x}^{2}+\xi_{y}^{2}-\omega^{2}\right) \bar{F}^{y}-\left(\beta^{2}-1\right)\left(\xi_{x}^{2} \bar{F}^{y}+\xi_{x} \xi_{y} \bar{F}^{x}\right)\right. \\
& \left.\left.+\frac{2 i c_{1}^{2}}{\beta^{2}}\left(\beta^{2}-1\right) \alpha \bar{T} \xi_{y}\left(\xi_{x}^{2}+\xi_{y}^{2}-\omega^{2}\right)\right\} \cdot \exp \left[-i\left(x \xi_{x}+y \xi_{y}+\omega \tau\right)\right]\right\} \\
& \cdot\left\{\left(\xi_{x}^{2}+\xi_{y}^{2}-\beta^{2} \omega^{2}\right)\left(\xi_{x}^{2}+\xi_{y}^{2}-\omega^{2}\right)\right\}^{-1} \cdot d W \\
\tau^{x x}= & \frac{-i \rho}{(2 \pi)^{\frac{3}{2}}} \int_{W_{3}}\left\{\beta^{2}\left(\xi_{x}^{2}+\xi_{y}^{2}-\omega^{2}\right)\left(\xi_{x} \bar{F}^{x}+\nu \xi_{y} \bar{F}^{y}\right)-\left(\beta^{2}-1\right)\left(\xi_{x}^{2}+\nu \xi_{y}^{2}\right)\right. \\
& \left.\left.\cdot\left(\xi_{x} \bar{F}^{x}+\xi_{y} \bar{F}^{y}\right)+\frac{2 i c_{1}^{2}}{\beta^{2}}\left(\beta^{2}-1\right) \alpha \bar{T}\left(\xi_{x}^{2}+\xi_{y}^{2}-\beta^{2} \omega^{2}\right)\left[(v-1) \xi_{x}^{2}+\omega^{2}\right]\right\}\right\} \\
& \cdot\left\{\left(\xi_{x}^{2}+\xi_{y}^{2}-\beta^{2} \omega^{2}\right)\left(\xi_{x}^{2}+\xi_{y}^{2}-\omega^{2}\right)\right\}^{-1} \cdot d W \\
\tau^{y y}=- & i \rho \frac{1}{(2 \pi)^{\frac{3}{2}}} \int_{W_{3}}\left\{\beta^{2}\left(\xi_{x}^{2}+\xi_{y}^{2}-\omega^{2}\right)\left(\nu \xi_{x} \bar{F}^{x}+\xi_{y} \bar{F}^{y}\right)-\left(\beta^{2}-1\right)\right. \\
& \left.\cdot\left(\nu \xi_{x}^{2}+\xi_{y}^{2}\right)\left(\xi_{x} \bar{F}^{x}+\xi_{y} \bar{F}^{y}\right)+\frac{2 i c_{1}^{2}}{\beta^{2}}\left(\beta^{2}-1\right) \alpha \bar{T}\left(\xi_{x}^{2}+\xi_{y}^{2}-\beta^{2} \omega^{2}\right)\right\} \\
& \left.\cdot\left[(\nu-1) \xi_{x}^{2}+\omega^{2}\right]\right\} \cdot \exp \left[-i\left(x \xi_{x}+y \xi_{y}+\omega \tau\right)\right] \\
& \cdot\left\{\left(\xi_{x}^{2}+\xi_{y}^{2}-\beta^{2} \omega^{2}\right) \cdot\left(\xi_{x}^{2}+\xi_{y}^{2}-\omega^{2}\right)\right\}^{-1} \cdot d W \\
\tau^{x y}= & \frac{-i \rho}{\beta^{2}} \frac{1}{(2 \pi)^{\frac{3}{2}}} \int_{W_{3}}\left\{\beta^{2}\left(\xi_{x}^{2}+\xi_{y}^{2}-\omega^{2}\right)\left(\xi_{y} \bar{F}^{x}+\xi_{x} \bar{F}^{y}\right)-2\left(\beta^{2}-1\right) \xi_{y} \xi_{x}\right. \\
& \left.\left.\cdot\left(\xi_{x} \bar{F}^{x}+\xi_{y} \bar{F}^{y}\right)+\frac{2 i c_{1}^{2}}{\beta^{2}}\left(\beta^{2}-1\right) \alpha \bar{T}\left(\xi_{x}^{2}+\xi_{y}^{2}\right)\left(\xi_{x}^{2}+\xi_{x}^{2}-\beta^{2} \omega^{2}\right)\right]\right\} \\
& \cdot \exp \left[-i\left(x \xi_{x}+y \xi_{y}+\omega \tau\right)\right] \cdot\left\{\left(\xi_{x}^{2}+\xi_{y}^{2}-\beta^{2} \omega^{2}\right) \cdot\left(\xi_{x}^{2}+\xi_{y}^{2}-\omega^{2}\right)\right\} \cdot d W
\end{array}\right\}
$$

where $W$ is the $\xi_{x} \xi_{y} \omega$ space and $d W=d \xi_{x} d \xi_{y} d \omega$.

Now, from the set of relations $\tau^{x x}+\tau^{y y}, \tau^{x x}-\tau^{y y}$, normal stresses $\tau^{x x}$ and $\tau^{y y}$ can be obtained easily.

\section{Solution of the Problem}

We consider the solution of the equation of motion when the time-dependent body force ${ }^{[3]}$ and temperature $T$, acting at the origin in the direction of $x$-increasing, varies harmonically with time period $\frac{2 \pi}{\rho}$. For such case we may write

$$
\begin{gathered}
X=\frac{F^{x}}{\rho} \delta(x) \delta(y) \delta(t) \\
T=\frac{T_{0}}{2 \mu} \delta(x) \delta(y) \delta(t)
\end{gathered}
$$

which gives us for $\bar{X}$ and $\bar{T}$, the relation

$$
\bar{X}=\frac{F^{x} c_{1}}{\rho(2 \pi)^{\frac{3}{2}}}
$$




$$
\bar{T}=\frac{T_{0} c_{1}}{2 \mu(2 \pi)^{\frac{3}{2}}}
$$

since $\delta(t)=c_{1} \delta(\tau)$. Here we have chosen for the shake of simplicity $F^{x}=X, F^{y}=0$. We shall adopt the usual notation for this problem, i.e.

$$
v^{x}=u_{T}, \quad v^{y}=v_{T}, \quad \tau^{x x}=\left(\sigma_{x}\right)_{T}, \quad \tau^{y y}=\left(\sigma_{y}\right)_{T}, \quad \tau^{x y}=\left(\tau_{x y}\right)_{T} .
$$

Putting $F^{x}=X, F^{y}=0$ and using the usual notations we get from (11) and (12),

$$
\left.\begin{array}{rl}
u_{T}= & \frac{1}{c_{1}^{2}(2 \pi)^{\frac{3}{2}}} \int_{W_{3}}\left[\frac{\beta^{2}\left(\xi_{x}^{2}+\xi_{y}^{2}-\omega\right)-\left(\beta^{2}-1\right) \xi_{x}^{2}}{\left(\xi_{x}^{2}+\xi_{y}^{2}-\beta^{2} \omega^{2}\right)\left(\xi_{x}^{2}+\xi_{y}^{2}-\omega^{2}\right)} \bar{X}\right. \\
& \left.+\frac{2 i c_{1}^{2}}{\beta^{2}} \frac{\left(\beta^{2}-1\right) \alpha \xi_{x}}{\left(\xi_{x}^{2}+\xi_{y}^{2}-\beta^{2} \omega^{2}\right)} \bar{T}\right] \cdot \exp \left\{-i\left(x \xi_{x}+y \xi_{y}+\omega \tau\right)\right\} \cdot d W
\end{array}\right\}
$$

Rewriting $u_{T}$ and $v_{T}$ we get,

$$
\left.\begin{array}{rl}
u_{T}= & \frac{1}{c_{1}^{2}(2 \pi)^{\frac{3}{2}}} \int_{W_{3}}\left[\frac{\bar{X}}{\xi_{x}^{2}+\xi_{y}^{2}}\left\{\frac{\xi_{x}^{2}}{\xi_{x}^{2}+\xi_{y}^{2}-\omega^{2}}+\frac{\beta^{2} \xi_{y}^{2}}{\xi_{x}^{2}+\xi_{y}^{2}-\beta^{2} \omega^{2}}\right\}\right. \\
& \left.\left.+2 i \frac{\mu}{\rho}\left(\beta^{2}-1\right) \alpha \bar{T} \frac{\xi_{x}}{\left(\xi_{x}^{2}+\xi_{y}^{2}-\beta^{2} \omega^{2}\right)}\right] \cdot \exp \left\{-i\left(x \xi_{x}+y \xi_{y}+\omega \tau\right)\right\} \cdot d W\right\} \\
v_{T}= & \frac{1}{c_{1}^{2}(2 \pi)^{\frac{3}{2}}} \int_{W_{3}}\left[\frac{\bar{X} \xi_{x} \xi_{y}}{\xi_{x}^{2}+\xi_{y}^{2}}\left\{\frac{1}{\xi_{x}^{2}+\xi_{x}^{2}-\omega^{2}}-\frac{\beta^{2}}{\xi_{x}^{2}+\xi_{y}^{2}-\beta^{2} \omega^{2}}\right\}\right. \\
& \left.-2 i \frac{\mu}{\rho}\left(\beta^{2}-1\right) \alpha \bar{T} \frac{\xi_{y}}{\left(\xi_{x}^{2}+\xi_{y}^{2}-\beta^{2} \omega^{2}\right)}\right] \cdot \exp \left\{-i\left(x \xi_{x}+y \xi_{y}+\omega \tau\right)\right\} \cdot d W
\end{array}\right\}
$$

Subsitiuting the value of $\bar{X}$ and $\bar{T}$ form (18) and (19) into (20) and (21), we get

$$
\left.\begin{array}{rl}
u_{T}= & -\frac{1}{8 \pi^{3}} \frac{F c_{1}}{\mu \beta^{2}}\left(\frac{\partial^{2} I_{1}}{\partial x^{2}}+\beta^{2} \frac{\partial^{2} I_{2}}{\partial y^{2}}\right) \\
& -\frac{1}{8 \pi^{3}} \frac{\left(\beta^{2}-1\right)}{\mu \beta^{2}} \alpha T_{0} c_{1} \frac{\partial}{\partial x}\left(\frac{\partial^{2} I_{2}}{\partial x^{2}}+\frac{\partial^{2} I_{2}}{\partial y^{2}}\right)
\end{array}\right\}
$$


where

$$
\begin{aligned}
& I_{1}=\int_{W_{3}} \frac{\exp \left\{-i\left(x \xi_{x}+y \xi_{y}+\omega \tau\right)\right\}}{\left(\xi_{x}^{2}+\xi_{y}^{2}\right)\left(\xi_{x}^{2}+\xi_{y}^{2}-\omega^{2}\right)} \cdot d W \\
& I_{2}=\int_{W_{3}} \frac{\exp \left\{-i\left(x \xi_{x}+y \xi_{y}+\omega \tau\right)\right\}}{\left(\xi_{x}^{2}+\xi_{y}^{2}\right)\left(\xi_{x}^{2}+\xi_{y}^{2}-\beta^{2} \omega^{2}\right)} \cdot d W
\end{aligned}
$$

Putting $x=r \cos \theta, y=r \sin \theta, \xi_{x}=\rho \cos \phi, \xi_{y}=\rho \sin \phi$ in (24) and (25).

After making necessary substitution and then integrating we find that $\frac{\partial I_{2}}{\partial r}$ is a function of $r$ only, and that ${ }^{[4]}$

$$
\begin{aligned}
\frac{\partial I_{2}}{\partial_{r}} & =-\frac{4 \pi^{2}}{\beta} \int_{0}^{\infty} \frac{\sin \left(\frac{\rho \tau}{\beta}\right) J_{1}(\rho r)}{\rho} d \rho \\
& = \begin{cases}-\frac{4 \pi^{2} \tau}{\beta^{2} r}, & (\tau \leq \beta r) \\
-\frac{4 \pi^{2}}{\beta^{2} r}\left\{\tau-\sqrt{\left(\tau^{2}-\beta^{2} r^{2}\right)}\right\}, & (\tau \geq \beta r)\end{cases}
\end{aligned}
$$

A similar expression can be obtained for $\frac{\partial I_{1}}{\partial r}$ by putting $\beta=1$ in equation (26). Substituting these values into equation (22) and (23) we obtain the formula

$$
\begin{aligned}
& u_{T}=\left\{\begin{array}{cl}
0, & (r>\tau) \\
\frac{F c_{1}}{2 \pi \mu \beta^{2}}\left[\frac{x^{2}}{r^{2}}\left(\tau^{2}-r^{2}\right)^{-\frac{1}{2}}+\frac{x^{2}-y^{2}}{r^{4}}\left(\tau^{2}-r^{2}\right)^{\frac{1}{2}}\right], & \left(\tau^{\prime}<r<\tau\right) \\
\frac{F c_{1}}{2 \pi \mu \beta^{2}}\left[\frac{x^{2}}{r^{2}}\left(\tau^{2}-r^{2}\right)^{-\frac{1}{2}}+\frac{\beta y^{2}}{r^{2}}\left(\tau^{\prime 2}-r^{2}\right)^{-\frac{1}{2}}\right. & \\
\left.+\frac{x^{2}-y^{2}}{r^{4}}\left\{\left(\tau^{2}-r^{2}\right)^{\frac{1}{2}}-\beta\left(\tau^{\prime 2}-r^{2}\right)^{\frac{1}{2}}\right\}\right] & \\
+\frac{T_{0} \alpha c_{1}}{2 \pi \mu \beta^{3}}\left(\beta^{2}-1\right) x\left(\tau^{\prime 2}-r^{2}\right)^{-\frac{3}{2}}, & (r<\tau) \\
&
\end{array}\right. \\
& v_{T}= \begin{cases}0, & (r>\tau) \\
\frac{F c_{1}}{2 \pi \mu \beta^{2}} \frac{x y}{r^{2}}\left\{\left(\tau^{2}-r^{2}\right)^{-\frac{1}{2}}+\frac{2}{r^{2}}\left(\tau^{2}-r^{2}\right)^{\frac{1}{2}}\right\}, & \left(\tau^{\prime}<r<\tau\right) \\
-\frac{T_{0} c_{1}}{2 \pi \mu} \frac{\alpha}{\beta^{3}}\left(\beta^{3}-1\right) y\left(\tau^{\prime 2}-r^{2}\right)^{\frac{-3}{2}} & \\
+\frac{x y}{r^{2}} \frac{F c_{1}}{2 \pi \mu \beta^{2}}\left\{\left(\tau^{\prime 2}-r^{2}\right)^{-\frac{1}{2}}-\beta\left(\tau^{\prime 2}-r^{2}\right)^{-\frac{1}{2}}\right\} & \\
\left.+\frac{2}{r^{2}}\left[\left(\tau^{2}-r^{2}\right)^{\frac{1}{2}}-\beta\left(\tau^{\prime 2}-r^{2}\right)^{\frac{1}{2}}\right]\right\}, & \left(r<\tau^{\prime}\right)\end{cases}
\end{aligned}
$$


In these formula $\tau=c_{1} t, \tau^{\prime}=c_{2} t=\frac{c_{1} t}{\beta}$.

These determine the components of displacement vector.

Differentiating $u_{T}$ and $v_{T}$, and substituting these values in (4) we obtain,

$$
\frac{\tau \beta^{2}\left[\left(\sigma_{x}\right)_{T}-\left(\sigma_{y}\right)_{T}\right]}{F c_{1}}= \begin{cases}\frac{T_{0} \alpha}{F} \cdot \frac{\left(\beta^{2}-1\right)}{\beta}\left\{2\left(\tau^{\prime 2}-r^{2}\right)^{-\frac{3}{2}}+3 r^{2}\left(\tau^{\prime 2}-r^{2}\right)^{-\frac{5}{2}}\right\} & \\ +\left(\tau^{2}-r^{2}\right)^{-\frac{3}{2}}-\frac{3 y^{2}}{r^{2}}\left\{\left(\tau^{2}-r^{2}\right)^{-\frac{3}{2}}-\beta\left(\tau^{\prime 2}-r^{2}\right)^{-\frac{3}{2}}\right\} & \\ -\frac{2}{r^{4}}\left(x^{2}-3 y^{2}\right)\left\{\left(\tau^{2}-r^{2}\right)^{-\frac{1}{2}}-\beta\left(\tau^{\prime 2}-r^{2}\right)^{-\frac{1}{2}}\right\} & \\ -\frac{4}{r^{6}}\left(x^{2}-3 y^{2}\right)\left\{\left(\tau^{2}-r^{2}\right)^{\frac{1}{2}}-\beta\left(\tau^{\prime 2}-r^{2}\right)^{\frac{1}{2}}\right\}, & \left(r<\tau^{\prime}\right), \\ \frac{x^{2}-y^{2}}{r^{2}}\left(\tau^{2}-r^{2}\right)^{-\frac{3}{2}}-\frac{2\left(x^{2}-3 y^{2}\right)}{r^{4}}\left(\tau^{2}-r^{2}\right)^{-\frac{1}{2}} & \\ -\frac{4}{r^{6}}\left(x^{2}-3 y^{2}\right)\left(\tau^{2}-r^{2}\right)^{\frac{1}{2}}, & \left(\tau^{\prime}<r<\tau\right), \\ 0, & (r>\tau)\end{cases}
$$

$$
\frac{2 \pi \beta^{2}\left[\left(\sigma_{x}\right)_{T}-\left(\sigma_{y}\right)_{T}\right]}{\left(\beta^{2}-\right) c_{1} F}= \begin{cases}x\left(\tau^{2}-r^{2}\right)^{-\frac{3}{2}}, & (r<\tau) \\ 0, & (r>\tau),\end{cases}
$$

$$
\frac{2 \pi \beta^{2}\left(\tau_{x y}\right)_{T}}{y c_{1} F}= \begin{cases}\frac{\beta^{2}\left(\tau^{\prime 2}-r^{2}\right)^{-\frac{3}{2}}+\frac{2 x^{2}}{r^{2}}\left\{\left(\tau^{\prime 2}-r^{2}\right)^{-\frac{3}{2}}-\beta\left(\tau^{\prime 2}-r^{2}\right)^{-\frac{3}{2}}\right\}}{r^{4}}\left\{\left(\tau^{2}-r^{2}\right)^{-\frac{1}{2}}-\beta\left(\tau^{\prime 2}-r^{2}\right)^{-\frac{1}{2}}\right\} & \\ -\frac{4}{r^{6}}\left(x^{2}-3 y^{2}\right)\left\{\left(\tau^{2}-r^{2}\right)^{-\frac{1}{2}}-\beta\left(\tau^{\prime 2}-r^{2}\right)^{-\frac{1}{2}}\right\}, & \left(r<\tau^{\prime}\right), \\ \frac{2 x^{2}}{r^{2}}\left(\tau^{2}-r^{2}\right)^{-\frac{3}{2}}+\frac{2\left(y^{2}-3 x^{2}\right)}{r^{4}}\left(\tau^{2}-r^{2}\right)^{-\frac{1}{2}}, & \\ +\frac{4}{r^{6}}\left(x^{2}-3 y^{2}\right)\left(\tau^{2}-r^{2}\right)^{\frac{1}{2}}, & \left(\tau^{\prime}<r<\tau\right), \\ 0 & (r>\tau)\end{cases}
$$

From (29) and (30) principal stresses $\left(\left(\sigma_{x}\right)_{T},\left(\sigma_{y}\right)_{T}\right)$ can be determined easily.

From the expressions it is clear that the distribution is propagated outwards from the centre with velocities $c_{1}$ and $c_{2}=\frac{c_{1}}{\beta}$. These waves are known in sismology as the $P$ waves, and $s$-waves, respectively (Bullen 1947, p.47). The wave fronts are circles, centre the origin and radii $\tau=c_{1} t, \tau^{\prime}=c_{2} t$. 


\section{References}

[1] A. C. Eringen and S. Suhubi, Elastodynamics, Chap. 5, Academic Press, 1975.

[2] A. E. Green and W. Zerna, Theoretical Elasticity, O. U. P., 1975.

[3] G. Eason, J. Fulton and I. N. Sneddon, Generation of waves in solids, Phil. Trans. Roy. Soc. (A) 248, 575, (1956).

[4] I. N. Sneddon, Fourier Transform, Mc-Graw-Hill Pub., New York, 1961.

[5] S. Timoshenko and J. M. Goodier, Theory of Elasticity, 2nd Edn., Mc-Graw Hill Pub., New York, 1951.

Computer Centre, North Bengal University, Darjeeling, West Bengal, pin-734430, India.

Department of Mathematics, North Bengal University, Darjeeling, West Bengal, pin-734430, India. 